\title{
Kant y el conocimiento de sí mismo
}

\section{Efraín Lazos}

\author{
No tengo una idea de mí mismo; \\ ni la que consiste en una falta de idea \\ de mí mismo. \\ Soy un nómada de la conciencia de mí mismo.
}

Fernando Pessoa

\section{I}

$\mathrm{D}$

espués de doscientos años de interpretación, uno debería tener una buena razón para discutir ciertos temas y pasajes de la Crítica de la razón pura. Eso es particularmente aplicable a la discusión kantiana sobre el conocimiento del yo, que cubre pasajes de primera importancia en la obra, tales como la "Deducción trascendental de las categorías" y "Los paralogismos de la razón pura". Sin embargo, creo tener una razón. Con su reciente reelaboración de la noción de "segunda naturaleza" - la idea de que mediante la educación y el aprendizaje los seres humanos normales pueden moldear su intelecto de modo que atiendan demandas de la razón-, John McDowell ha argumentado que, de haber contado Kant con ella, su brillante concepción de la experiencia humana "no sólo se habría liberado del efecto distorsionante del marco en el que trata de expresarla, sino que también permitiría que la conexión entre la autoconciencia y la conciencia del mundo, que figura de un modo equívoco en su pensamiento, tomara una forma satisfactoria". ${ }^{1}$ Este reproche tiene el eco reconocido de la lectura de la filosofía teórica kantiana por parte de P. F. Strawson, en su célebre libro, Los límites del sentido. Según esta interpretación, que McDowell suscribe, Kant percibió correctamente que la posibilidad de la experiencia de objetos y la posibilidad de la autoadscripción de experiencias por parte de un sujeto son interdependientes. El problema, no obstante, es que este sujeto de experiencias no es para Kant el sujeto ordinario, $i$. $e$. una primera persona que es a la vez una tercera persona, sino un sujeto extraordinario, o "trascendental", que mantiene una posición privilegiada respecto de la propia experiencia y cuya continuidad temporal en el mundo se reduce a la continuidad

\footnotetext{
${ }^{1}$ John McDowell, Mind and World. Harvard, Universidad de Harvard, 1994, p. 99.
} 
formal de un "mero punto de vista". ${ }^{2}$ En esa medida, el acierto de Kant acerca de la interdependencia mencionada se ve opacado por su imposibilidad de dar cuenta del hecho de que el sujeto que se atribuye experiencias puede identificarse a sí mismo con un ser vivo particular, con "una presencia corporal en el mundo". El veredicto de McDowell es claro: la percepción correcta de Kant podría tomar una forma satisfactoria "sólo si pudiera conceder el hecho de que un sujeto que tiene pensamientos e intenciones es un animal vivo", pero la ausencia de una noción seria de segunda naturaleza le impide hacerlo. ${ }^{3}$

Éste es, me parece evidente, un asunto interpretativo que debe considerarse con detenimiento. No amerita disputa la idea de que la noción de segunda naturaleza está ausente en la primera Crítica $;{ }^{4}$ tampoco está en discusión que el objeto de la crítica filosófica que ofrece Kant en pasajes como "Los paralogismos" y la "Refutación al idealismo" es lo que se podría llamar el modelo cartesiano de la mente. ${ }^{5}$ Creo, sin embargo, que vale la pena investigar si, y en qué medida, Kant tiene éxito en esa empresa. Porque si Kant logra en efecto "exorcizar las tentaciones cartesianas acerca del yo" ello quiere decir que, en este punto, no requiere introducir la noción de segunda naturaleza. Esto, casi sobra decirlo, no dejará de hacer dicha noción menos valiosa, y tal vez no hará que la filosofía teórica de Kant sea más persuasiva ante los ojos contemporáneos. Pero puede contribuir a esclarecer un problema interpretativo interesante por sí mismo.

En este trabajo me concentraré en la sección sobre "Los paralogismos de la razón pura", sobre todo, aunque no exclusivamente, en la versión de la segunda edición de la Crítica de la razón pura. Dejaré de lado la crítica al idealismo problemático que se encuentra en el cuarto paralogismo de la primera edición, correspondiente, en la segunda, a la "Refutación al idealismo".

${ }^{2}$ Ibid., p.100; P. F. Strawson, The Bounds of Sense. Londres, Menthuen, 1966, p.164.

${ }^{3}$ En este punto la lectura de McDowell se separa de la de Strawson. Para este último el culpable ausente es el concepto primitivo de persona. Para Strawson, como para McDowell, el culpable presente es, desde luego, esa forma de subjetivismo extremo que Kant llama idealismo trascendental.

${ }^{4}$ Esta afirmación es correcta, a pesar de que la noción asociada de Bildung ciertamente aparece de modo importante en la filosofía crítica de Kant, en sus escritos de filosofía práctica, y en especial en la Crítica de la facultad de juzgar. Trad. introd. y notas de Pablo Oyarzún R. Caracas, Monte Ávila, 1991. Cf. Crítica del juicio. Trad. y pról. de Manuel García Morente. México, Porrúa, 1980, parág. 83.

${ }^{5}$ G. Ryle, The Concept of Mind. Nueva York, Barnes \& Noble, 1949. Esta afirmación puede ser disputada si uno concuerda con Jonathan Bennett, La "Crítica de la razón pura" de Kant. II. La dialéctica.Trad. de Julio César Armero. Madrid, Alianza,1981, p. 81. De cualquier forma, la afirmación no pretende tener alcances históricos, esto es, no disputa si el modelo corresponde íntegramente con lo que Descartes afirmó. 
Elaboro, antes, una reconstrucción del modelo cartesiano de la mente, que propongo consignar en cuatro tesis centrales. La exposición que ofrezco no recorre paso a paso los textos kantianos, pero tampoco renuncia a la exégesis de este o aquel pasaje de la obra.

\section{II}

Según el modelo cartesiano de la mente, hay dos historias que contar acerca de una persona: la historia pública, accesible a cualquiera, que incluye datos de una persona en un momento determinado, información que en principio es accesible a todos los que quieran y puedan buscarla y encontrarla; y, la historia privada, que constituye, en este modelo, algo así como el coto vedado de la persona misma. De esta segunda historia hay que observar que no se trata ahí de información que no sea, de hecho, conocida por otros (e.g., lo que se reserva como información privilegiada), sino del conocimiento de determinadas experiencias que, por su propio peso, otros no pueden conocer. Hay entonces un sentido débil y un sentido fuerte de la palabra privado. El primero es inclusivo y se toca en realidad con lo aludido en la historia pública: se trata de datos ocultos que la persona no revela o no quiere revelar, pero que cualquiera, dadas las circunstancias, podría conocer; sin embargo, el sentido en el que se usa el término en el mencionado modelo es restrictivo de modo enfático, y se encuentra pretendidamente en expresiones como "sólo yo sé lo que siento" o "tú no sabes lo que yo siento". Este modelo ofrece, en breve, una manera de entender lo mental como algo privado de modo enfático, $i$. e. como aquello de lo cual el único testigo confiable es la persona misma que se lo atribuye.

Una manera tal vez más canónica de presentar el modelo es diciendo que las dos vidas que cada persona, según hemos dicho, lleva, son o corresponden a dos modos de ser o de existir. Al primero se le suele llamar físico, corporal o material (también se le llama "lo externo") y al segundo, mental, intelectual o psicológico ("lo interno"). Y se suele complementar esta presentación diciendo que es característico de lo físico suceder en el espacio y en el tiempo, mientras que lo mental sucede en el tiempo, pero no en el espacio. Si bien se puede dar cuenta de la interacción entre los cuerpos en términos causales, no hay una conexión causal directa entre lo que ocurre en una mente y lo que ocurre en otra. De ahí un posible eslogan del modelo, "sólo los cuerpos se tocan, no las mentes". Cada cual tiene acceso directo a su propia vida mental, que transcurre, por así decirlo, en su isla personal, pero no puede tener acceso a las mentes de otros, a lo que otros creen, quieren, juzgan, sino de manera indirecta, derivada. Por lo demás, no es necesario para el cartesiano afirmar que cada cual tiene acceso directo a todos los episodios de su vida mental 
-puede conceder que hay sucesos ocultos de su biografía mental (Freud)-, pero al menos algunos de tales episodios, e. $g$. los presentes, son transparentes a la persona en cuya mente ocurren. ${ }^{6}$

Esta transparencia de los propios estados mentales indica, además, que cuando de hecho conozco lo que pienso, juzgo, creo, no lo hago porque acuda al mundo "externo" o porque otros me lo digan, sino en virtud de un acto de introspección: miro dentro de mí y sé con certeza qué contiene mi mente. Un ser omnisciente, si lo hubiera, podría conocer directamente qué ocurre en la mente de alguien en un momento dado de su vida, sin tener que conocer nada acerca del mundo en el que la persona se encuentra. Para nosotros, sin embargo, sólo es posible una transparencia circunscrita a nuestra propia vida mental, las mentes de otros están irremediablemente ocultas. ${ }^{7}$ De este modo, la historia que de cada cual pueden contar otros es completamente independiente de la historia que cada cual cuenta de sí mismo: lo que sólo yo puedo saber, sólo yo puedo contar.

Las siguientes tesis asociadas caracterizan el modelo:

$T_{1}$. Cada cual tiene autoconciencia epistémica inmediata, esto es, conocimiento con certeza de los propios estados subjetivos.

$T_{2}$. Los objetos de tal autoconciencia inmediata son los contenidos privados de la conciencia individual; como consecuencia de lo cual,

$T_{3}$. Cada cual puede tener sólo un conocimiento inferencial, y falible, de los contenidos de conciencia de otros

$T_{4}$. El conocimiento de la propia experiencia interna es el fundamento de la experiencia de un mundo objetivo. ${ }^{8}$

Lo que en $T_{1}$ queda expresado es que cuando un sujeto $A$ se adscribe asimismo una determinada experiencia y, $A$ puede siempre decir "yo y" con verdad, solamente en virtud de tener la experiencia que de hecho tiene. Si estoy deprimido, por ejemplo, no tiene sentido decir: "Ésta es una depresión, pero ¿̇soy yo quien está deprimido?" ( $\mathrm{O}$ : "recuerdo el dolor de muelas, pero ¿soy yo quien lo recuerda?, ¿era yo quien lo tenía?) Este elemento de indubitabilidad que se encuentra en las autoadscripciones de experiencias se

${ }^{6}$ Un ejemplo notorio de la posición en este punto se encuentra en Bertrand Russell, "On the Nature of Acquaintance (1914)", en Logic and Knowledge. Londres, Allen \& Unwin, 1977, pp. 162, 166-167.

${ }^{7}$ Cf. M. Cavell, The Psycholanalytic Mind. Harvard, Universidad de Harvard, 1993, pp. $12-13$

${ }^{8}$ Existe un correlato lingüístico de este modelo contra el cual dirige Wittgenstein su célebre "argumento del lenguaje privado". Ver R. A. Noë, "Did Kant Anticipate Wittgenstein's Private Language Argument?”, en Kant-studien, vol. 82, núm. 3, 1991, p. 270. 
toma en el modelo como evidencia del carácter epistémico especial de las mismas: todas las experiencias que un sujeto tiene pueden expresarse en juicios infalibles, juicios que no pueden no ser verdaderos. La indubitabilidad, en el sentido de infalibilidad, está estrechamente conectada con el elemento de privacidad enfática del modelo $\left(T_{2}\right)$ y contrasta con el conocimiento que cada cual puede tener de objetos de la experienca ordinaria y de otras mentes $\left(T_{3}\right)$. Y dado que la propia experiencia interna es indubitable, hay grandes prospectos para usarla como la base inamovible de todo conocimiento de objetos $\left(T_{4}\right)$.

\section{III}

Tiene Kant motivos importantes para atacar el modelo cartesiano de la mente. Señalo dos. Primero, si el modelo es correcto, Kant tendría que enfrentar un desafío a su idea de que todo conocimiento requiere de la intervención de las categorías: del "yo", cada cual tendría precisamente el tipo de conocimiento que no está mediado por las categorías del entendimiento. En segundo término, y de manera notoria, si el idealismo trascendental de Kant (como la teoría de que el espacio y el tiempo y todos los fenómenos en ellos no son sino representaciones) puede usarse para probar que la autoconciencia es sólo posible si es posible la conciencia de objetos del mundo externo, entonces no sólo el cartesianismo estaría suficientemente refutado, sino que cualquier duda acerca de un compromiso del idealismo trascendental con el cartesianismo quedaría eliminada. Kant desarrolla este último argumento como "la única prueba posible de la realidad de los objetos del sentido externo" sobre todo en el cuarto paralogismo de la primera edición de la Crítica y en la "Refutación del idealismo", en la segunda, y su consideración será objeto de otro trabajo. El ataque que Kant monta en los tres primeros paralogismos, y en la versión correspondiente a la segunda edición, tiene como cometido general enfrentar el desafío mencionado en primer lugar.

Kant ataca, en su crítica a la psicología racional, una serie de argumentos, llamados paralogismos, que toman como punto de partida la pretendida certeza con la que cada cual puede adscribirse experiencias a sí mismo. Todos ellos se proponen establecer que cada cual sabe con certeza que existe como una sustancia pensante, como un sujeto de pensamientos y experiencias, que es inmaterial y simple, y que persiste con entera independencia del cuerpo.

En ambas ediciones de la obra, Kant sostiene que el primer paralogismo ejemplifica una falacia que se encuentra en la premisa menor de todos los paralogismos. En la formulación de la primera edición: 
Sustancia es aquello cuya representación constituye el sujeto absoluto de nuestros juicios, aquello que no puede, por tanto, ser empleado como determinación de otra cosa.

Yo, en cuanto ser pensante, soy el sujeto absoluto de todos mis juicios posibles, pero esa representación de mí mismo no puede ser usada como predicado de otra cosa.

Consiguientemente, yo, en cuanto ser pensante (alma) soy sustancia. [A 348] ${ }^{9}$

Con distintos énfasis, Kant propone que la falacia se genera al usar el psicólogo racional términos con distinto sentido en las premisas mayor y menor. La primera edición sugiere que los términos se usan en sentido "trascendental" en la primera premisa, y en sentido empírico en la premisa intermedia y en la conclusión (A 402).

Una interpretación de lo que Kant quiere decir cuando indica que una categoría, como la de sustancia, se emplea trascendentalmente, es que se usa en forma independiente de las condiciones que permitirían establecer si hay objetos que se pueden subsumir bajo ese concepto. Y la categoría se usaría empíricamente si se le refiere a un "esquema" que permitiera establecer qué objetos determina. La invalidez del argumento radicaría en lo siguiente. Si la categoría "pura" es la categoría sin su correspondiente esquema (que permitiría determinar un objeto de la intuición) o, dicho de otro modo, si la categoría esquematizada contiene una necesaria determinación de la categoría "pura", entonces podría afirmarse que las sustancias empíricas son un subconjunto de las sustancias trascendentales, y que, en correspondencia, los sujetos empíricos son un subconjunto de los sujetos trascendentales. Pero, aunque, trascendentalmente, todos los sujetos, podrían ser sustancias, ello no permite concluir que un sujeto empírico particular pertenece al subconjunto de las sustancias empíricas. ${ }^{10}$

Otra manera de formular el objeto de la crítica de Kant en estos pasajes es la afirmación de que toda experiencia es siempre experiencia personal. Esto es, toda experiencia es algo tal que necesariamente alguien puede decir de ella "yo y" (donde "y" simboliza cualquier juicio de experiencia). Formulada de esta manera, la posición que Kant ataca tiene un lado que difícilmente se disputaría, y puede tomarse como la verdad trivial de que toda experiencia es experiencia de alguien. Lo que resulta problemático es considerar

${ }^{9}$ E. Kant, Crítica de la razón pura. Trad. de Pedro Ribas. Madrid, Alfaguara, 1978. $C f:$ : B410-11.

${ }^{10}$ Este argumento se encuentra desarrollado en P. Kitcher, "Kant"s Paralogisms", en The Philosophical Review, vol. 91, núm. 4, 1982, pp. 515-547. 
que esa verdad trivial como que aporta conocimiento objetivo de aquello a lo que se refiere la representación "yo y". Éste es el énfasis general que pone Kant cuando diagnostica, en la segunda edición de la Crítica, el error en el que se precipita el psicólogo racional, el filósofo cartesiano. Las proposiciones del psicólogo racional acerca del alma -que es sustancia, que es la misma en tiempos distintos, que es simple y que es inmaterial- son meros juicios analíticos que se derivan trivialmente de lo que contiene "la conciencia de mí mismo en el pensamiento en general", esto es, se derivan de lo que Kant ha llamado la unidad trascendental de la apercepción. No obstante, el psicólogo racional pretende que esas proposiciones sean juicios sintéticos con valor objetivo y que expresen, en tanto tales, un conocimiento efectivo de la naturaleza del yo. Lo que sólo pertenece a "una discusión [Erörterung] lógica del pensamiento" se considera, ilusoriamente, como "una determinación metafísica del objeto" (B 409).

Lo anterior muestra con claridad que la crítica a las ilusiones de la psicología racional está montada sobre una célebre idea kantiana que aparece en la "Deducción trascendental", la idea de que "el 'yo pienso' tiene que poder acompañar todas mis representaciones" (B 131). En primera instancia, esta afirmación puede tomarse como una manera rebuscada de expresar la idea, casi trivial, de que todos mis juicios son míos. Pero pierde ese aire de trivialidad cuando se expresa, en general, como que todo juicio, si es tal, debe poder atribuírsele a un sujeto. En otra formulación, esto quiere decir que si, para cualquier juicio y que se tome, debe ser posible atribuir y a algún sujeto, entonces debe ser posible que alguien forme una proposición verdadera de tipo "yo pienso que y". Bien mirada, ésta es una manera alternativa de concebir lo contenido en la tesis $T_{1}$ del modelo cartesiano de la mente, $i$. $e$., la tesis de que cuando un sujeto $A$ se adscribe a sí mismo una determinada experiencia y, $A$ puede siempre decir "yo y" con verdad. Y es alternativa porque no es en virtud de tener la experiencia, que de hecho tiene, que el sujeto en cuestión puede formular tal juicio con verdad, sino sólo en virtud de lo que significa representar, pensar, juzgar. Si no se aceptara tal principio, que Kant denomina el principio de la necesaria unidad de la apercepción, la consecuencia sería el sinsentido, o, como lo expresa él mismo, "sería representado en mí algo que no podría ser pensado, lo que equivale a decir que la representación, o bien sería imposible, o bien no sería nada para mí". Kant indica en la continuación de este pasaje que la afirmación de que la autoconciencia debe ser posible es en realidad un principio idéntico, una verdad analítica. ${ }^{11}$

11 "El principio de la necesaria unidad de la apercepción es, a su vez, idéntico y constituye, por tanto, una proposición analítica [...]" (B 135). Esto permite también 
No considero que la afirmación de Kant en el sentido de que tal principio contiene, también, una síntesis, sea una objeción a lo que vengo diciendo. El principio de la posibilidad de la autoconciencia presupone, en efecto, que lo diverso sea sintetizado en la intuición. Creo que esto significa simplemente que algo debe ser representado, debe haber ya un juicio, para que la representación pueda adjudicarse a un sujeto. Pero una vez producida la representación, la autoconciencia se sigue analíticamente de lo que significa representar un objeto. Si bien el asunto merece, desde luego, un tratamiento más extenso del que aquí puedo ofrecer, hay que indicar además que el principio de la necesaria unidad de la apercepción, en su aspecto de unidad sintética, le permite a Kant, contra Hume, sancionar el hecho de que no podemos atribuir estados de conciencia (concíbanse ya como juicios, ya como intuiciones) si no están éstos conectados entre sí. En otros términos, sólo podemos atribuir contenido a juicios o intuiciones si los consideramos como parte de un sistema de estados de conciencia que mantienen relaciones de mutua dependencia. Cuando Kant habla de una relación de síntesis entre representaciones, al menos parte de lo que afirma es que las representaciones que ocurren en tiempos distintos dependen mutuamente en términos de contenido -dependen unas de otras para representar aquello que representan. Así, una de las tesis contenidas en la noción de la unidad trascendental de la apercepción es que una condición indispensable para la posibilidad de la experiencia es que las representaciones que ocurren en tiempos distintos formen parte de un sistema interdependiente de representaciones. ${ }^{12}$

\section{IV}

La interpretación que he presentado del diagnóstico kantiano de las ilusiones de la psicología racional parece concordar con la descripción general que hace Strawson del pasaje correspondiente en la Crítica. Para Strawson, Kant pretende deslindarse del modelo cartesiano mostrando que, si bien es necesario que cada cual pueda percatarse de sus propios estados de conciencia, ello no significa que podamos inferir las propiedades que el psicólogo racional atribuye al yo. Según Strawson, Kant detecta claramente, en contraste con el psicólogo racional, que no hay criterios de identidad que estén involucrados en la autoadscripción inmediata de experiencias: "cuando un

explicar una versión de la ilusión de la psicología racional que aparece en la primera edición de la obra. Dicho sucintamente, se confunde el hecho de que la representación "yo pienso" puede ser un aspecto invariable en todos nuestros juicios, con la idea, distinta, de que puede intuirse el yo como algo permanente. $C f$. A 350.

${ }^{12}$ Vid. P. Kitcher, op. cit., pp. 224-226. 
hombre (un sujeto de experiencia) se adscribe a sí mismo un estado de conciencia presente o directamente recordado, no requiere usar criterio alguno de identidad personal para justificar su empleo del pronombre 'yo' para referirse al sujeto de tal experiencia". ${ }^{13}$ Esto quiere decir que, cuando alguien se adscribe experiencias a sí mismo, no realiza individuación alguna de un sujeto. Eso explica por qué no tiene sentido dudar, cuando me duele la cabeza, si es a mí a quien le duele la cabeza; y da cuenta de por qué el psicólogo racional toma erróneamente la autoadscripción inmediata de experiencias, en donde la duda es ininteligible, como algo que ofrece al filósofo un conocimiento especial del sujeto al que se refiere la primera persona en los juicios que expresan dicha adscripción.

Sin embargo, si la interpretación que he ofrecido del texto de Kant es correcta, Strawson está equivocado en este punto. Aquello a lo que Strawson se refiere al hablar de "autoadscripciones" no constituye, de hecho, nada parecido a verdades analíticas. Expresiones tales como "me duele la cabeza" o "yo recuerdo a tu tía" serían ejemplos de juicios empíricos cuya "verdad" no parece tener nada que ver con el significado de "representación" o "juicio". Si mi lectura es adecuada, Kant se interesa en negar que lo que se pueda derivar del principio de unidad analítica de la apercepción tenga un contenido epistémico, y es justamente ésa su manera de negar un status epistémico especial a las conclusiones del filósofo cartesiano: no brindan ellas una forma particularmente efectiva de conocimiento del yo porque no brindan conocimiento de nada. Tales juicios, en suma, son sólo expresiones de una ilusión.

Strawson, inmiscuido en su propio proyecto de metafísica descriptiva, está preocupado por la cuestión de cómo preservar un contenido para las autoadscripciones inmediatas, a pesar de que en ellas el pronombre personal se usa, según su propia doctrina, sin criterios empíricos que permitan individuar su referente. ${ }^{14}$ Su respuesta es bien conocida. Terceras personas pueden de hecho identificar al sujeto mediante el uso de criterios de identidad personal. En la práctica, los vínculos entre el uso del pronombre "yo" sin criterios y los criterios públicos para identificar personas no están truncados. Y esto se debe a que tanto las autoadscripciones como las adscripciones de experiencias a otros se hacen a propósito del mismo tipo de individuos, a saber, personas.

${ }^{13} C f$. P. F. Strawson, op. cit., p.165; R. A. Noë, op. cit., p. 272.

${ }^{14}$ Esta idea presupone, si estoy en lo correcto, la aceptación de que usar una palabra requiere la aplicación de criterios determinados. El Wittgenstein de las Investigaciones filosóficas invita a sospechar de ello, en su célebre tratamiento de la noción de "seguir una regla”. Cf. Investigaciones filosóficas. México/Barcelona, 1988, parág. 201-206; ver también: R. A. Noë, op. cit., pp. 279-283. 
McDowell acepta a pie juntillas esta lectura strawsoniana de Kant. De ahí que continuamente demande de la filosofía de Kant algo que no le puede dar, $i$. $e$. la continuidad material del sujeto de la experiencia. Si partimos de que el error en el que incurre el psicólogo racional es que omite el hecho de que el "yo" de las autoadscripciones de experiencias puede usarse sin criterios de identidad, no nos queda sino pensar que la teoría kantiana de la unidad de la apercepción no logra exorcizar del todo las tentaciones del modelo cartesiano de la mente. Por ello, como lo expresa McDowell, "más vale que el 'yo pienso' que tiene que poder acompañar todas mis representaciones sea sólo una abstracción de la persistencia sustancial ordinaria de un sujeto de la experiencia vivo". ${ }^{15}$ Si no es así, peor para él.

Sin embargo, si estoy en lo correcto la teoría kantiana no lo requiere para deslindarse exitosamente del cartesianismo. En lo que toca a la tesis $\left(T_{1}\right)$ de la autoconciencia inmediata en el modelo cartesiano de la mente, el punto nodal del argumento es que la posibilidad de un conocimiento inmediato de los propios estados de conciencia depende de la posibilidad de tener experiencia de aquello que no es parte de los contenidos de la propia conciencia. En otros términos, la concepción que restringe la experiencia epistémicamente relevante a los datos privados de la conciencia $\left(T_{2}\right)$ pierde sentido en el contexto de una estructura más amplia dentro de la cual debe ser posible aplicar exitosamente conceptos de un mundo objetivo espacio-temporal. La posibilidad de nuestro conocimiento de objetos que no son los contenidos de nuestros propios estados mentales se demuestra como una condición de posibilidad del conocimiento inmediato de los contenidos de nuestra propia conciencia. Kant se compromete, es cierto, con una versión de la falibilidad de la adscripción de estados de conciencia a otros $\left(T_{3}\right)$, una especie de teoría analógica de las otras mentes. ${ }^{16}$ Piensa que nuestro conocimiento de otras mentes es indirecto, mediado por una transferencia de nuestra autoconciencia a otros seres, $y$, por tanto, falible. Bien mirado, el argumento general permite, finalmente, rechazar la idea $\left(T_{4}\right)$ de que la experiencia interna es el fundamento de la experiencia de un mundo objetivo: si sólo es posible concebir la experiencia humana en el contexto de un sistema interconectado de representaciones, y si el contenido de tales representaciones presupone la determinación de objetos espacio-temporales, mecánicamente conectados, entonces no es posible afirmar que la experiencia interna tiene un status epistémico privilegiado que funda la experiencia de un mundo objetivo.

${ }^{15} \mathrm{~J}$. McDowell, op. cit., p. 103.

${ }^{16}$ A 346/B 405 